\title{
Im Spiegel des Klimawandels - Phosphor und Stickstoff in Böden Schleswig-Holsteins bei steigenden Lufttemperaturen
}

\author{
Andreas Rinker · Frauke Deunert · Winfried Schröder
}

Eingereicht: 18. September 2008 / Akzeptiert: 15. Januar 2009/Online veröffentlicht: 31. März 2009

(C) Springer-Verlag 2009

Zusammenfassung Ziel und Hintergrund Der globale Klimawandel wirkt sich regional differenziert auf einzelne Ökosystem-Kompartimente aus. Aufgrund der zentralen regulatorischen Bedeutung des Stoffhaushalts terrestrischer Ökosysteme war es das Ziel dieser Untersuchung im Auftrag des Landesamtes für Natur und Umwelt SchleswigHolstein, den Austrag von Phosphor (P) und Stickstoff (N) aus Böden Schleswig-Holsteins unter dem heutigen und zukünftigen Klima zu berechnen.

Methoden Methodischer Mittelpunkt der Untersuchungen ist das Modellsystem Wasmod, mit dem der wassergebundene Stofftransport in Böden und an der Bodenoberfläche für Bodenprofile, Ackerflächen oder Wassereinzugsgebiete berechnet werden kann. Die Wasmod-Berechnungen dieser Studie erfolgten auf der Grundlage von Daten der Agrarstatistik, des Deutschen Wetterdienstes (DWD), der RemoKlimaprognosen sowie der Reichsbodenschätzung. Wasserund Stoffbilanzen wurden für typische Kombinationen von acht Bodensubstraten, vier Grundwasserflurabstand-Stufen, neun Anbauvarianten und des Klimas auf landwirtschaftlich genutzten Flächen berechnet. Die Modellrechnungen erfolgten für den Zeitraum vom 1. Januar 1975 bis zum 31. Dezember 2004 (30 Jahre) für die Klimastandorte Erfde und Lensahn des DWD. Die Klimaszenarien wurden für den Zeitraum 1971-2000 (Referenz) sowie für die Jahre 20712100 (Remo-Szenarien B1 und A1B) ausgewertet. Alle Berechnungen erfolgten mit und ohne Boden-Drainage.

A. Rinker $\cdot$ F. Deunert

DigSyLand - Institut für Digitale Systemanalyse \& Landschaftsdiagnose, Zum Dorfteich 6, 24975 Husby, Deutschland

W. Schröder $(\square)$

Lehrstuhl für Landschaftsökologie, Hochschule Vechta,

Postfach 1553, 49464 Vechta, Deutschland

E-Mail: wschroeder@iuw.uni-vechta.de
Ergebnisse Die Ergebnisse umfassen Angaben über den Niederschlag, die Interzeption, die Evapotranspiration, das Sickerwasser, den Drainage-Abfluss sowie ausgewählte N-Bilanzen (Eintrag, Sickerwasseraustrag, Mineralisation, Denitrifikation, Nitrifikation, Volatilisierung, Drainage-Abfluss) sowie P-Bilanzen (Eintrag, Mineralisation, Auswaschung und Anteil an organischem, sorbiertem, gelöstem und fixiertem P). Die Ergebnisse der Simulationen weisen eine hohe Differenzierung der Merkmalskombinationen auf. Die Evapotranspiration steigt in den Klimaszenarien B1 und insbesondere A1B deutlich an. Die Sickerwassermenge ist im Szenario B1 im Vergleich zum Referenzzeitraum sehr hoch, da auch mehr Niederschläge fallen. A1B weist dagegen ähnliche Resultate wie das Referenzszenario trotz höherer Niederschläge auf, denn die höhere Verdunstungsrate kompensiert den Niederschlagszuwachs. Das Klimaszenario B1 weist die höchsten P- und N-Austräge auf. Das Klimaszenario A1B ist den Resultaten des Referenzlaufes sehr ähnlich. Die N-Austräge unter Ackernutzung steigen im Klimaszenario B1 stark an. Die höchsten Austräge (136 kg N/ha) wurden für intensiv genutzte Weidestandorte berechnet. Das B1-Szenario weist die höchsten P-Austräge auf, A1B rangiert unterhalb denen des Referenzzeitraums. Die N-Austräge der sandigen Standorte sind im Mittel des Verhältnisses Einträge/Austräge höher als bei lehmigen Standorten, absolut aber geringer. Räumlich differenzierend wirkt sich hierbei der Grundwasserflurabstand aus. Die Ergebnisse der Simulationen bezüglich der gasförmigen N-Verluste weisen auf den sandigen Standorten erwartungsgemäß kaum Denitrifikationsverluste auf. Bei den lehmigen Standorten können die Denitrifikationsverluste auf $41 \mathrm{kgN} /$ ha im Jahr ansteigen. Die höchsten Mineralisationsraten sind im Szenario A1B zu erwarten. Das Szenario B1 weist im Vergleich zum Referenzszenario erhöhte Temperaturen bei hohen Niederschlägen und wenig Trockenperioden auf, sodass eine Abnahme der Mineralisationsleitung auf 
diesen Standorten berechnet wurde. Drainagen verringern die N- und P-Auswaschung aus Äckern, hingegen steigt der P-Output aus drainiertem Grünland.

Diskussion Eine Diskussion der Ergebnisse kann nur methoden- und datenkritisch sowie wissenschaftstheoretisch erfolgen, aufgrund fehlender weiterer Untersuchungen jedoch nicht im Vergleich mit anderen Befunden. Wegen der mittlerweile zwanzigjährigen Entwicklungszeit des Modellsystems Wasmod und seiner breiten Validierung in der Ökosystemforschung, in Fallstudien sowie im Umwelt-Monitoring kann davon ausgegangen werden, dass der Wasser- und Stoffhaushalt der Böden zuverlässig modelliert wird. Eine große Herausforderung bleibt die Regionalisierung der Modellergebnisse durch die Verknüpfung von Daten der Reichsbodenschätzung mit Daten aus dem Klima- und Phänologie-Monitoring. Schlussfolgerungen Grundlagenforschung in Form der Ökosystemforschung erweist sich insofern als lohnend, als man dadurch jetzt in der Lage ist, validierte Modelle wie Wasmod für Prognosen des Stoffhaushalts in Böden unter veränderten Klimabedingungen berechnen zu können. Entsprechende Grundlagen fehlen z. B. bei der Frage nach dem Zusammenhang zwischen Klimaänderungen und Gesundheit insbesondere für die prognostische Modellierung des Risikos von Krankheiten, deren Pathogene und Überträger (Vektoren) in ihrer Entwicklung und ihrer geografischen Verbreitung stark von der Lufttemperatur abhängig sind.

Empfehlungen und Ausblick Grundlagenforschung im Bereich der Ökologie und Umweltmedizin wäre die beste Vorbereitung auf Umweltkrisen und sollte nicht abgewickelt, sondern fortentwickelt werden. Hierbei müsste konsequent darauf geachtet werden, dass Daten aus Forschungsprojekten und Monitoring-Programmen so dokumentiert werden, dass sie über Metadaten-Informationssysteme recherchiert und zusammengeführt werden können. Die Auswirkungen des Klimawandels auf den Stoffhaushalt in Böden sollten bundesweit regionalisiert werden. Hierfür sollten anhand der landschaftsökologischen Gliederung Deutschlands sowie anhand von Klimadaten und pflanzenphänologischen Daten des Deutschen Wetterdienstes und der internationalen phänologischen Gärten repräsentative Modellierungsräume ausgewählt und untersucht werden. Weiterhin wird dieser Ansatz mit der prädiktiven Kartierung des Risikos von durch Vektoren übertragenen Krankheiten von Menschen und Tieren verknüpft.

Schlüsselwörter Böden $\cdot$ Klimawandel $\cdot$ Modellsystem Wasmod $\cdot$ Phosphat $\cdot$ Schleswig-Holstein $\cdot$ Stickstoff

\section{$P$ and $\mathbf{N}$ in soils of Schleswig-Holstein reflecting increasing air temperatures}

Abstract Background, aim, and scope The ecological effects of the global climate change differ regionally. Due to the ecological significance of soils in terrestrial ecosystems the aim of this study on behalf of the State Agency for Nature and Environment of Schleswig-Holstein was to calculate the output of nitrogen and phosphate from soils in Schleswig-Holstein (Germany) given the current and predicted air temperatures.

Methods The calculations were performed by the Water and Substance Modelling System (WASMOD). This complex simulation tool can be used for modelling the fluxes of water and dissolved substances through single soil columns or for fields and watersheds. In this study the calculations rely on data collected from several sources such as agricultural statistics, the German Weather Service DWD, results from the high resolution regional climate model REMO as well as from the German soil inventory "Reichsbodenschätzung". The WASMOD calculations were performed for representative combinations of ecological characteristics such as soil texture, distance to the ground water table, drainage, cultivation, and climate. The computations refer to three periods: 1975 to 2004 according to the measurements of two meteorological stations (DWD), and in terms of REMO modelling 1971 to 2000 (reference), and 2071 to 2100 according to the IPCC scenarios B1 and A1B.

Results The WASMOD results encompass precipitation, interception, evaporation, seepage water, drainage, nitrogen balances (input, mineralisation, denitrification, nitrification, output, volatilisation, and drainage), and phosphate balances (input, mineralisation, output, and percentage of organic, sorbed, dissolved and immobile P). According to the combinations of influencing factors the computed results vary spatially: Given the scenarios B1 and A1B the evaporation is expected to increase. Compared to the reference period the B1 seepage will be high and associated with high precipitation. Nevertheless, due to increasing evaporation A1B is similar to the reference period despite higher precipitation. The B1 scenario showed the highest outputs of $\mathrm{N}$ and $\mathrm{P}$. The results for A1B were found to be very similar with those for the reference period. The $\mathrm{N}$ output from fields significantly increase in B1. The highest outputs were estimated for intensively grazed feedlots. For B1 the highest $\mathrm{P}$ outputs were computed whereas the A1B estimations were lower than the results referring to the reference period. In terms of the input/output ratio the $\mathrm{N}$ output from sandy soils will be higher than those from loamy soils while the contrary holds true for the absolute discharge. These findings will vary spatially with the distance of the soil surface from the groundwater table. The expected output by denitrification is negligible whereas from loamy soils such $\mathrm{N}$ losses can account for $41 \mathrm{~kg} / \mathrm{ha}$. The mineralisation was expected at maximum in A1B. Compared with the reference period B1 will have higher air temperatures and more precipitation but less droughts. Consequently, for these sites reduced mineralisation was estimated. Draining will reduce the $\mathrm{N}$ 
and $\mathrm{P}$ output from fields and enhance the P discharge from grassland, respectively.

Discussion The WASMOD modelling results could not be compared to findings of similar studies because such could not be investigated. Thus, the discussion had to focus on the data and methods used for the computations, and the results could not be validated empirically. Nevertheless, from many case studies, ecosystem research and environmental monitoring projects which have been published during the last 20 years the computations of this investigation could be classified as, at least, plausible. A major challenge of further investigations will be the regionalisation if the WASMOD results by the combination of high resolution soil data with such on plant phenology.

Conclusions Basic research as for instance long-term ecosystem research should be regarded as essential precondition for the development of prognostic models as presented by the example of WASMOD. Such research could be proved as worthwhile in face of problems as represented in this study. Regarding the global change related risk of vector-associated diseases the predictive power of ecological models must be broadened and refined.

Recommendations and perspectives Ecosystem research and environmental epidemiology should be coordinated much closer. This research must be attended by a professional documentation of research and monitoring results by use of metadata-based and GIS-based networking of local databases. The approach of the investigation presented should be applied to representative ecoregions of Germany. To reach this, modelling areas will be selected in terms of an ecological land classification and extensive data sets from meteorological and phenological monitoring networks. Additionally, the approach will be extended by the predictive mapping of vector-associated diseases.

Keywords Climate change - Nitrogen · Phosphate · Schleswig-Holstein $\cdot$ Soils $\cdot$ Water and substance modelling system (WASMOD)

\section{Ziel und Hintergrund}

Es wird mittlerweile nicht mehr darüber gestritten, ob ein Klimawandel eingetreten ist. Vielmehr geht es darum, seine Ausprägung und seine ökologischen Konsequenzen räumlich differenziert zu bestimmen, um regionalisierte Anpassungsstrategien zu entwickeln. Die räumliche Differenzierung der ökologischen Folgen des Klimawandels kann nicht für komplexe Ökosystemprozesse erfolgen, sondern muss schutzgutspezifisch anhand von aussagekräftigen Indikatoren erfolgen. Deren Aussagekraft sollte durch ökologische Grundlagenforschung untermauert sein, und sie sollten möglichst flächendeckend quantifiziert wer- den können. Für die acht Schutzkategorien Atmosphäre, Kryosphäre, marine, terrestrische und limnische Ökosysteme, Landwirtschaft, Wirtschaft sowie menschliche Gesundheit wurden 22 Indikatoren identifiziert (EEA 2004). Die Klimawandelindikatoren für terrestrische Ökosysteme sind: Avifauna, Kohlenstoffspeicherung sowie Zusammensetzung, Arealentwicklung und Phänologie von Pflanzen. Unberücksichtigt bleibt dabei der Stoffhaushalt der Böden, obwohl diese das regulatorische Hauptkompartiment terrestrischer Ökosysteme und damit neben dem Standortklima die zweite essenzielle Randbedingung der Primärproduktion sind (Fränzle et al. 1993). Dieser Erkenntnis entsprach die Verknüpfung von bio- und geowissenschaftlichen Forschungsansätzen in der Ökosystemforschung, deren programmatische Grundlegung eng mit Ellenberg et al. (1978) verknüpft ist. In dem Projekt „Ökosystemforschung im Bereich der Bornhöveder Seenkette" war die Erfassung von Wasser- und Stoffflüssen in und zwischen Ökosystemen ein Arbeitsschwerpunkt (Fränzle 1998). Ein umfangreiches Messprogramm lieferte Daten zur Bilanzierung der N-Einträge in die einzelnen Systemkompartimente sowie zur Quantifizierung der für den Haushalt wichtigen Transport- und Transformationsprozesse. Diese Untersuchungen wurden in terrestrischen (Acker, Grünland, Wälder), semiterrestrischen (Feuchtgrünland, Erlenbruch, Niedermoorgebiete) und in limnischen Ökosystemen durchgeführt. Ziel war es u.a., auf mehreren räumlichen Ebenen (lokal, regional) ökosystemare Prozesse zu erfassen und deren Beeinflussung durch Menschen abzuschätzen. Die mittel- und langfristige Wirkung von anthropogen bedingten Stoffeinträgen in Ökosysteme unterschiedlicher Empfindlichkeit war hierbei ein wichtiger Aspekt. Da nicht alle relevanten Prozesse durch Messungen erfasst werden konnten oder der Messaufwand für manche ökosystemaren Prozesse zu hoch und zu kostenträchtig ist, stellte die Entwicklung und Anwendung von Simulationsmodellen wie Wasmod einen wichtigen Teilbereich der Arbeiten dar (Fränzle et al. 2008). Ausreichend validierte Modelle bieten die Möglichkeit, Prozesse zeitlich und räumlich zu extrapolieren (Matthies et al. 2006). Mit dem Modellsystem WASMOD (Reiche 1991) lässt sich die Wasser- und Stoffdynamik für einzelne Bodenprofile, für Ackerflächen und für Wassereinzugsgebiete berechnen. In Abhängigkeit von den jeweiligen Merkmalsausprägungen von Vegetation, Relief und Böden sowie agrarwirtschaftlicher Nutzung erfolgt die Prozessquantifizierung räumlich hierarchisiert für die Vegetationsdecke, die Bodenoberfläche, den durchwurzelten Boden sowie die wasserungesättigte und -gesättigte Zone. Vertikale und laterale Transportprozesse werden pro Zeitschritt nacheinander simuliert. Besonders berücksichtigt werden die von Mikroorganismen gesteuerten Umsetzungsprozesse und ihre spezifischen Wechselbeziehungen zum Kohlenstoff- und N-Haushalt. 
Ziel dieser Untersuchung im Auftrag des Landesamtes für Natur und Umwelt Schleswig-Holstein war die Simulation folgender Teilprozesse in ausgewählten Böden Schleswig-Holsteins und an ihrer Oberfläche unter heutigem und vorausgesagtem Klima: Wasserdynamik (mit Interzeption, Evapotranspiration, Infiltration), Dränabfluss, Grundwasserabfluss und Grundwasserflurabstand, vertikaler Transport gelöster Stoffe (mit Aufnahme durch Pflanzenwurzeln), Ad- und Desorptionsdynamik von Stoffen, vertikaler Wärmetransport, Kohlenstoffumsetzung (Humifizierung, Mineralisation mit Quantifizierung der $\mathrm{CO}_{2}$-Freisetzung), mikrobiell gesteuerte N-Umsetzung (Ammonifizierung, Nitrifizierung, Denitrifikation, Immobilisierung), gasförmige $\mathrm{NH}_{4}-\mathrm{N}$-Verluste, lateraler Transport gelöster Stoffe durch die wassergesättigte Zone, Transport gelöster Stoffe mit dem Dränwasser, langfristige Veränderungen der Wasserleitfähigkeit und des Wasserhaltevermögens in Abhängigkeit von der Humusdynamik sowie Quantifizierung der $\mathrm{N}$ - und C-Akkumulation unterschiedlicher Pflanzenkompartimente.

\section{Material und Methoden}

\subsection{Modellstruktur WASMOD}

Fink und Kralisch (2005), Kralisch et al. (2003, 2005), Müller et al. (2006) sowie Rinker (2001) beschreiben den Aufbau, die Funktionen und die Validierung des Simulationsmodells Wasmod. Das Hauptprogramm regelt die Einund Ausgabe sowie die Prozesssteuerung. Die Prozesse werden entsprechend ihrem Raum- und Zeitbezug in spezifischen Zeitintervallen aufgerufen. Beispielsweise werden die sich langfristig verändernden Merkmale wie die pF- und kf-Funktionen nur jährlich modifiziert. Hingegen werden schnell ablaufende Prozesse wie die Bodenwasserbewegung im Minuten- bzw. Stundenrhythmus für die vier Ökosystem-Kompartimente Pflanzen, Boden, Grundwasser und Vorfluter berechnet.

\subsection{Daten}

Für die Berechnungen wurden betriebswirtschaftliche und betriebsübergreifende Daten zur Agrarstruktur, zur Bodennutzung, zur Ernte, zum ökologischen Landbau (LWK-SH 2001a-c; MLLT 2002; StA-HH-SH 2004a-d, 2005a-c), zu Düngerichtwerten (LWK-SH 2003) und langjährige Datensätze der DWD-Klimastationen Erfde und Lensahn mit Tageswerten zu Minimum und Maximum der Luftemperatur, der Niederschlagsmenge und der Luftfeuchtigkeit zwischen dem 1. Januar 1975 und dem 31. Dezember 2004 verwendet. Die Modellierungen des N- und des P-Haushalts erfolgten zudem für den Referenzzeitraum (1971-2000) und die Remo-Szenarien A1B und B1 des IPCC (2001) für die Jahre 2071-2100. das A1B-Szenario geht von starkem wirtschaftlichen Wachstum und intensiver Nutzung fossiler und nicht-fossiler Brennstoffe aus. Das Szenario B1 unterstellt Dienstleistungsgesellschaften mit nachhaltiger Ressourcennutzung.

Aus rund 500.000 Datensätzen der Reichsbodenschätzung wurden nach häufigkeitsstatistischen und bodenhydrologischen Kriterien 3.300 relevante Bodenartenkombinationen identifiziert. Die Zuordnung von $\mathrm{pH}-$ Wertstufen zu den Bodenarten erfolgte in Anlehnung an die anzustrebenden pH-Zielwerte (LWK-SH 2003). Die Informationen zur Landnutzung entstammen den Agrarstatistiken. In Expertengesprächen wurden typische Fruchtfolgen naturräumlich und substratspezifisch differenziert. Für die Grünlandnutzung wurden Schnitt, Weide und Mähweidenutzung verschiedener Intensitäten ebenfalls substratspezifisch bestimmt, wobei den Nutzungshäufigkeiten die entsprechende Düngung in $\mathrm{kgN} / \mathrm{ha}$ zugeordnet wurde. Die absoluten Zahlen orientieren sich dabei nach LWK-SH (2003). Für die Düngung der Ackerkulturen wurden ebenfalls die Empfehlungen von LWK-SH (2003) angewendet. Dabei wird aus den Standorteigenschaften das mögliche Ertragsniveau bestimmt, dem zu den Kardinalpunkten der Pflanzenentwicklung (Vegetationsbeginn, Schossen, Spätdüngung zum Ährenschieben) N-Gaben zugeordnet werden. Die Phaseneintrittstermine errechnet WASMOD anhand der Kulturpflanzen-Phänologie und dem Witterungsverlauf. Bei der Modellierung der P-Düngung konnte im Gegensatz zu N nicht auf Ergebnisse von Bodenuntersuchungen zurückgegriffen werden, da diese nicht veröffentlicht werden. Daher wurde angenommen, dass für die modellierten Szenarien eine mittlere Gehaltsklasse mit guter Verfügbarkeit entsprechend der Versorgungsstufe $\mathrm{C}$ vorlag und erhalten werden sollte. Aus Bodenart, Fruchtfolge und den Annahmen zur Intensität der landwirtschaftlichen Nutzung wurden Ertragsstufen bestimmt. Für jeweils vier Ertragserwartungsstufen der häufigsten Fruchtarten wurde je nach Versorgungsstufe der Nährstoffbedarf gemäß MLLT (2002) und StA-HHSH (2004a-d, 2005a-c) zugeordnet. Den Hauptbodenarten Sand, Sand über Lehm, sandiger Lehm und Lehm wurden Ertragserwartungen zugewiesen, in denen sich das Ertragspotenzial der Standorte widerspiegelte. Den Sandböden wurden hierbei die geringsten und den sandigen Lehmen die höchsten Ertragserwartungen zugesprochen. Die P-Düngung des Gründlands wird nach der gleichen Vorgehensweise berechnet. Zusätzlich wurde zwischen Weiden und Wiesennutzung und Nutzungsintensität unterschieden. Aus der räumlichen Verknüpfung der oben zusammenfassend beschriebenen Merkmale ergeben sich 325 häufigkeitsstatistisch relevante Kombinationen, zu denen Eingabedateien für die Berechnungen mit Wasmod angelegt wurden (Abschn. 2.3). 


\subsection{Berechnungen}

Die Daten (Abschn. 2.1) wurden für die Berechnungen des $\mathrm{N}$ - und des P-Haushaltes entsprechend der Modellstruktur in mehreren Eingabedateien abgelegt. Diese werden im ASCIIFormat für einen Simulationslauf eingelesen, die Ergebnisse gehen in demselben Format als Input in den nächsten Berechnungsgang. Die Bodendatei beinhaltet in 15 Tiefenstufen differenzierte bodenphysikalische und bodenchemische Kennwerte sowie standortabhängige Angaben wie u. a. Flächengröße, Nutzungsvariante, Abstand zum Vorfluter und Hangneigung. Am Ende einer Simulationsperiode wird die Bodendatei von WASMOD neu geschrieben. Dabei werden u. a. die aktuelle Bodenfeuchte, der $\mathrm{C}_{\text {org }}$-Gehalt und der Grundwasserstand in der Datei mit den simulierten Werten gespeichert. Die CN-Datei enthält für jede der 15 Tiefenstufen der Böden Anfangswerte für unterschiedliche N-Formen. Die P-Datei enthält für jede der 15 Tiefenstufen der Böden Anfangswerte für unterschiedliche P-Fraktionen.

Die Kennzeichnung der Phänologie der Kulturarten erfolgt über die Durchwurzelungstiefe, den HAUDE-Faktor und den Blattflächenindex. Die Durchwurzelungstiefe bestimmt, aus welchen Tiefenstufen der Böden der Wasserentzug entsprechend der Transpirationsrate sowie die N-Aufnahme erfolgen. Bei einer angegebenen Wurzeltiefe von $\leq 5 \mathrm{~cm}$ findet keine N-Aufnahme statt. Der HAUDE-Faktor wird benötigt, um auf der Grundlage des Sättigungsdefizits der bodennahen Luftschicht eine vegetationsspezifische potenzielle Evapotranspirationsrate abzuschätzen. Die Angaben zum Blattflächenindex (LAI) werden für die Berechnung des Interzeptionsverlustes benötigt und dienen als Hilfsgröße bei der Abschätzung des Energieeintrages durch direkte Strahlung. Weiterhin enthalten die Phänologiedateien Angaben zum Nährstoffeintrag durch Düngung, zur pflanzenspezifischen Aufnahmekapazität und zu einzelnen landwirtschaftlichen Bearbeitungsmaßnahmen. WASMOD berücksichtigt neben dem in der Phänologiedatei angegebenen N-Eintrag einen zusätzlichen atmosphärischen Eintrag von $20 \mathrm{~kg} \mathrm{~N} / \mathrm{ha}$.

Die Klimadatei enthält tagesbezogene Angaben zur Minimum- und Maximumtemperatur, zum Niederschlag und zum Sättigungsdefizit (Haude) oder alternativ zur Global- strahlung (Turc-Wendling) der DWD-Stationen Erfde und Lensahn zwischen 1975 und 2004. Die Klimadaten der REMO-Referenz und -Szenarien (A1B und B1) wurden von der CERA-Datenbank heruntergeladen. Für die Jahresbilanzen wurden vier Modellläufe mit Klimadaten der DWD-Stationen Erfte und Lensahn und sechs Modellläufe mit Klimaszenarien ausgewertet:

1. Klimastation Erfde (1975-2004) Simulation ohne Drainagen,

2. dto. mit Drainagen,

3. Klimastation Lensahn (1975-2004) Simulation ohne Drainagen,

4. dto. mit Drainagen,

5. Klimaszenario Referenz (1971-2000) Simulation ohne Drainagen,

6. dto. mit Drainagen,

7. Klimaszenario A1B (2071-2100) Simulation ohne Drainagen,

8. dto. mit Drainagen,

9. Klimaszenario B1 (2071-2100) Simulation ohne Drainagen, 10. dto. mit Drainagen.

\section{Ergebnisse}

Die Sickerwassermenge ist im Szenario B1 im Vergleich zum Referenzzeitraum sehr hoch, da auch mehr Niederschläge fallen. A1B weist dagegen ähnliche Resultate wie das Referenzszenario auf. Die höhere Verdunstungsrate kompensiert den Niederschlagszuwachs (Tabelle 1).

Die höheren mittleren Niederschläge in Erfde führen zu größeren N-Austrägen $\left(\mathrm{NO}_{3}-\mathrm{N}\right)$ mit dem Sickerwasser (Tabelle 2). Mittelt man die Berechnungsergebnisse für Acker- und Grünland, ergeben sich N-Einträge von $157,5 \mathrm{~kg} \mathrm{NO}_{3}-\mathrm{N} /$ ha für die Station Erfde und Verluste von $73,5 \mathrm{~kg} \mathrm{NO}_{3}-\mathrm{N} / \mathrm{ha}$. Für Lensahn betragen die Austräge bei $64 \mathrm{~kg} \mathrm{NO}_{3}$-N/ha. Im Schnitt liegen die N-Einträge auf ackerbaulich genutzten Standorten mit $188 \mathrm{~kg} / \mathrm{ha}$ um $61 \mathrm{~kg} \mathrm{~N} / \mathrm{ha}$ über den Einträgen auf Grünlandstandorten. Dementsprechend sind die N-Verluste auf Ackerstandorten höher als bei

Tabelle 1 Wasserhaushaltsbilanzen

\begin{tabular}{lrcccccccccccccc}
\hline & \multicolumn{3}{c}{ Erfde (835) $^{\mathbf{a}}$} & \multicolumn{3}{c}{ Lensahn } & $(\mathbf{7 0 5})^{\mathbf{a}}$ & \multicolumn{3}{c}{ Referenz (838) $^{\mathbf{a}}$} & \multicolumn{3}{c}{ B1 (934) $^{\mathbf{a}}$} & \multicolumn{2}{c}{ A1B (876) $^{\mathbf{a}}$} \\
\hline Nutzung & Int & Eta & Sicker & Int & Eta & Sicker & Int & Eta & Sicker & Int & Eta & Sicker & Int & Eta & Sicker \\
Acker & 83 & 177 & 499 & 73 & 196 & 387 & 100 & 194 & 472 & 98 & 195 & 561 & 93 & 224 & 496 \\
Grünland & 154 & 195 & 444 & 135 & 214 & 329 & 175 & 209 & 413 & 187 & 212 & 489 & 175 & 245 & 424 \\
\hline
\end{tabular}

Int: Interzeption

Eta: aktuelle Evapotranspiration

Sicker: Sickerwasserverluste aus $1 \mathrm{~m}$ Tiefe

${ }^{\text {a Niederschlag }}$ 
Tabelle 2 N-Ein- und -Austräge

\begin{tabular}{|c|c|c|c|c|c|c|}
\hline & & $\operatorname{Erfde}(835)^{\mathrm{a}}$ & Lensahn $(705)^{a}$ & Referenz $(838)^{\mathrm{a}}$ & B1 $(934)^{\mathrm{a}}$ & A1B (876) \\
\hline Nutzung & Eintrag & \multicolumn{5}{|c|}{ Austrag $\left(\mathrm{NO}_{3}-\mathrm{N}\right)$} \\
\hline Acker & 188 & 89 & 79 & 87 & 100 & 89 \\
\hline Grünland & 127 & 58 & 49 & 62 & 64 & 57 \\
\hline
\end{tabular}

Ein- und Austräge in $\mathrm{kg} \mathrm{N} / \mathrm{ha}$

${ }^{a}$ Niederschlag in $\mathrm{mm}$

Tabelle 3 P-Ein- und -Austräge

\begin{tabular}{lcccccc}
\hline & & Erfde (835) & Lensahn (705) & Referenz (838) $^{\mathrm{a}}$ & B1 (934) $^{\mathrm{a}}$ & A1B (876) $^{\mathrm{a}}$ \\
\hline Nutzung & Eintrag & \multicolumn{5}{c}{ Austrag } \\
\hline Acker & 78 & 0,3137 & 0,2825 & 0,2870 & 0,3305 & 0,2721 \\
Grünland & 29 & 0,0825 & 0,0804 & 0,0739 & 0,0924 & 0,0664 \\
\hline
\end{tabular}

Ein- und Austräge in $\mathrm{kg} \mathrm{P} / \mathrm{ha}$

${ }^{a}$ Niederschlag in $\mathrm{mm}$

Grünlandnutzungen. Ähnliches gilt für das Referenzszenario. Für B1 sind steigende N-Austräge berechnet worden, insbesondere für die Ackerstandorte. Dagegen simuliert WASMOD für das Szenario A1B keine großen Unterschiede im Stoffaustrag gegenüber dem Referenzszenario.

Bei den P-Austrägen zeigt sich ein ähnliches Bild wie bei den N-Austrägen (Tabelle 3): Unter Grünlandnutzung wird wesentlich weniger $P$ über Düngung eingetragen und ausgewaschen. B1 weist wie bei den N-Austrägen die höchsten P-Verluste auf. A1B ist wiederum dem Referenzlauf sehr ähnlich.

Unter Getreidekulturen treten mit $98 \mathrm{~kg} \mathrm{NO}_{3}-\mathrm{N} / \mathrm{ha}$ und Jahr (Erfde) bzw. $89 \mathrm{~kg} \mathrm{NO}_{3}$-N/ha und Jahr (Lensahn) die höchsten N-Austräge auf (Tabelle 4). Auf den ohne Dün- gung genutzten Grünlandstandorten sind die geringsten $\mathrm{N}$-Austräge zu erwarten. Mit $122 \mathrm{~kg} \mathrm{NO}_{3}-\mathrm{N} / \mathrm{ha}$ und $\mathrm{Jahr}$ haben intensiv genutzte Weiden die höchsten Austräge. Die N-Austräge unter Acker steigen im Klimaszenario B1 stark an. Die höchsten Austräge mit $136 \mathrm{~kg} \mathrm{~N} / \mathrm{ha}$ wurden für intensiv genutzte Weidestandorte berechnet.

Unter Acker- und Grünlandnutzung wird in Erfde mehr P ausgetragen als in Lensahn (Tabelle 5). Die Ergebnisse des Referenzszenarios liegen in vergleichbarer Höhe des Klimastandorts Erfde. Für B1 wurden die höchsten Austräge berechnet. A1B weist geringere Austräge als der Referenzzeitraum auf.

Die sandigen Standorte (Sand, Sand über Lehm und lehmiger Sand) weisen im Vergleich zu den lehmigen Standor-

Tabelle 4 N-Ein- und -Austräge bei verschiedenen Nutzungen

\begin{tabular}{|c|c|c|c|c|c|c|c|}
\hline & & & $\operatorname{Erfde}(\mathbf{8 3 5})^{\mathrm{a}}$ & Lensahn $(705)^{a}$ & Referenz (838) ${ }^{a}$ & B1 $(934)^{a}$ & $\mathrm{~A} 1 \mathrm{~B}(876)^{\mathrm{a}}$ \\
\hline & Nutzung & Eintrag & & & Austrag $\left(\mathrm{NO}_{3}-\mathrm{N}\right)$ & & \\
\hline \multirow[t]{5}{*}{$\overline{\text { Acker }}$} & Mais & 186 & 90 & 80 & 94 & 102 & 90 \\
\hline & Raps & 191 & 81 & 69 & 78 & 95 & 81 \\
\hline & FF Getreide & 206 & 98 & 89 & 92 & 106 & 96 \\
\hline & FF bodenschonend & 206 & 97 & 88 & 92 & 106 & 96 \\
\hline & FF extensiv & 153 & 79 & 71 & 81 & 88 & 81 \\
\hline \multirow[t]{9}{*}{ Grünland } & Mahd intensiv & 260 & 88 & 69 & 94 & 95 & 81 \\
\hline & Mahd extensiv & 128 & 42 & 33 & 44 & 46 & 39 \\
\hline & Mahd ohne Düngung & 20 & 10 & 8 & 9 & 10 & 8 \\
\hline & Weide intensiv & 206 & 122 & 110 & 130 & 136 & 124 \\
\hline & Weide extensiv & 109 & 70 & 65 & 74 & 81 & 76 \\
\hline & Weide ohne Düngung & 20 & 15 & 12 & 15 & 16 & 15 \\
\hline & Mähweide intensiv & 254 & 100 & 88 & 111 & 112 & 95 \\
\hline & Mähweide extensiv & 124 & 58 & 48 & 62 & 66 & 58 \\
\hline & Mähweide ohne Düngung & 20 & 14 & 12 & 14 & 15 & 14 \\
\hline
\end{tabular}

Ein- und Austräge in $\mathrm{kg} \mathrm{N} / \mathrm{ha}$

a Niederschlag in mm 
Tabelle 5 P-Ein- und -Austräge bei verschiedenen Nutzungen

\begin{tabular}{|c|c|c|c|c|c|c|c|}
\hline & & & $\operatorname{Erfde}(835)^{\mathrm{a}}$ & Lensahn $(705)^{\mathrm{a}}$ & Referenz $(838)^{\mathrm{a}}$ & B1 (934) $)^{\mathrm{a}}$ & A1B (876) ${ }^{a}$ \\
\hline & Nutzung & Eintrag & & & Austrag & & \\
\hline \multirow[t]{5}{*}{$\overline{\text { Acker }}$} & Mais & 100 & 0,402 & 0,366 & 0,403 & 0,428 & 0,355 \\
\hline & Raps & 88 & 0,356 & 0,309 & 0,288 & 0,349 & 0,295 \\
\hline & FF Getreide & 81 & 0,329 & 0,302 & 0,301 & 0,356 & 0,286 \\
\hline & FF bodenschonend & 80 & 0,329 & 0,304 & 0,298 & 0,352 & 0,285 \\
\hline & FF extensiv & 42 & 0,153 & 0,133 & 0,146 & 0,168 & 0,139 \\
\hline \multirow[t]{9}{*}{ Grünland } & Mahd intensiv & 78 & 0,184 & 0,167 & 0,174 & 0,200 & 0,144 \\
\hline & Mahd extensiv & 41 & 0,089 & 0,082 & 0,072 & 0,092 & 0,065 \\
\hline & Mahd ohne Düngung & 0 & $<0,01$ & $<0,01$ & $<0.01$ & $<0,01$ & $<0,01$ \\
\hline & Weide intensiv & 34 & 0,131 & 0,134 & 0,123 & 0,167 & 0,124 \\
\hline & Weide extensiv & 18 & 0,066 & 0,075 & 0,056 & 0,080 & 0,060 \\
\hline & Weide ohne Düngung & 0 & $<0,01$ & $<0,01$ & $<0,01$ & $<0,01$ & $<0,01$ \\
\hline & Mähweide intensiv & 64 & 0,176 & 0,167 & 0,169 & 0,205 & 0,142 \\
\hline & Mähweide extensiv & 28 & 0,079 & 0,082 & 0,070 & 0,088 & 0,063 \\
\hline & Mähweide ohne Düngung & 0 & $<0,01$ & $<0,01$ & $<0,01$ & $<0,01$ & $<0,01$ \\
\hline
\end{tabular}

Ein- und Austräge in $\mathrm{kgP} / \mathrm{ha}$

${ }^{a}$ Niederschlag in $\mathrm{mm}$

ten (sandiger Lehm und Lehm im östlichen Hügelland) und Lehm (Marsch) geringere N-Einträge auf (Tabelle 6). Dementsprechend sind auch die absoluten Werte des N-Austrags niedriger. Die N-Austräge der sandigen Standorte sind jedoch im Mittel des Verhältnisses Einträge/Austräge höher als bei lehmigen Standorten, da durch die höhere Wasserleitfähigkeit der sandigen Bodenarten mehr Wasser versickert. Die Werte der N-Austräge in Lensahn sind wegen der hier um $130 \mathrm{~mm}$ niedrigeren mittleren Niederschlagssumme und des damit verbundenen geringeren Sickerwasserverlustes niedriger als in Erfde. Die geringsten N-Verluste mit $22 \mathrm{~kg} \mathrm{NO}_{3}-\mathrm{N} / \mathrm{ha}$ werden für Hochmoor unter Grünlandnutzung berechnet (Klimastation Lensahn). Mit $97 \mathrm{~kg} \mathrm{NO}_{3}-\mathrm{N} /$ ha werden die höchsten Austräge für Lehm unter Ackernutzung prognostiziert. Die Resultate des Referenzszenarios sind mit denen der Klimastation Erfde vergleichbar. B1 zeigt deutlich höhere Austräge, mit Ausnahme der Niedermoor- und Hochmoorstandorte, bei denen die Zunahme der Austräge weniger stark ausgeprägt ist. Die Werte für A1B (Spalte 8 in Tabelle 6) sind den für den Referenzzeitraum (Spalte 6 Tabelle 6) sehr ähnlich.

Für Erfde werden im Vergleich zu Lensahn durchweg höhere P-Austräge berechnet (Tabelle 7). Das Referenzsze-

Tabelle 6 Nach Bodenarten differenzierte N-Ein- und -Austräge mit dem Sickerwasser

\begin{tabular}{|c|c|c|c|c|c|c|c|}
\hline \multirow[b]{2}{*}{ Bodenart } & \multirow[b]{2}{*}{ Nutzung } & \multirow[b]{2}{*}{ Eintrag } & $\operatorname{Erfde}(835)^{a}$ & Lensahn $(705)^{a}$ & Referenz $(838)^{a}$ & B1 $(934)^{a}$ & A1B $(876)^{a}$ \\
\hline & & & \multicolumn{5}{|c|}{ Austrag $\left(\mathrm{NO}_{3}-\mathrm{N}\right)$} \\
\hline & Ackerland & 148 & 75 & 65 & 76 & 85 & 76 \\
\hline & Grünland & 125 & 66 & 56 & 65 & 72 & 65 \\
\hline \multirow[t]{2}{*}{ Sand über Lehm } & Ackerland & 170 & 93 & 82 & 91 & 102 & 91 \\
\hline & Grünland & 125 & 68 & 59 & 66 & 74 & 66 \\
\hline \multirow[t]{2}{*}{ Lehmiger Sand } & Ackerland & 164 & 84 & 75 & 81 & 92 & 81 \\
\hline & Grünland & 140 & 73 & 62 & 72 & 82 & 72 \\
\hline \multirow[t]{2}{*}{ Sandiger Lehm } & Ackerland & 199 & 90 & 80 & 91 & 102 & 88 \\
\hline & Grünland & 140 & 58 & 50 & 59 & 68 & 59 \\
\hline \multirow[t]{2}{*}{ Lehm (ÖH) } & Ackerland & 215 & 93 & 85 & 95 & 104 & 90 \\
\hline & Grünland & 144 & 52 & 43 & 51 & 59 & 57 \\
\hline \multirow[t]{2}{*}{ Lehm (Marsch) } & Ackerland & 215 & 97 & 85 & 95 & 108 & 93 \\
\hline & Grünland & 144 & 62 & 53 & 61 & 69 & 67 \\
\hline Niedermoor & Grünland & 100 & 47 & 41 & 44 & 49 & 48 \\
\hline Hochmoor & Grünland & 64 & 25 & 22 & 23 & 25 & 24 \\
\hline
\end{tabular}

ÖH: Östliches Hügelland; Ein- und Austräge in $\mathrm{kg} \mathrm{N} / \mathrm{ha}$

${ }^{a}$ Niederschlag in $\mathrm{mm}$ 
Tabelle 7 Nach Bodenarten differenzierte P-Ein- und -Austräge mit dem Sickerwasser

\begin{tabular}{|c|c|c|c|c|c|c|c|}
\hline \multirow[b]{2}{*}{ Bodenart } & \multirow[b]{2}{*}{ Nutzung } & \multirow[b]{2}{*}{ Eintrag } & Erfde $(835)^{\mathrm{a}}$ & Lensahn $(705)^{a}$ & Referenz $(838)^{\mathrm{a}}$ & B1 $(934)^{a}$ & A1B $(876)^{a}$ \\
\hline & & & \multicolumn{5}{|c|}{ Austrag } \\
\hline \multirow[t]{2}{*}{ Sand } & Ackerland & 64 & 0,147 & 0,141 & 0,114 & 0,160 & 0,131 \\
\hline & Grünland & 24 & 0,035 & 0,034 & 0,026 & 0,043 & 0,033 \\
\hline \multirow[t]{2}{*}{ Sand über Lehm } & Ackerland & 68 & 0,357 & 0,292 & 0,232 & 0,280 & 0,250 \\
\hline & Grünland & 24 & 0,117 & 0,090 & 0,060 & 0,077 & 0,058 \\
\hline \multirow[t]{2}{*}{ Lehmiger Sand } & Ackerland & 76 & 0,283 & 0,241 & 0,236 & 0,289 & 0,226 \\
\hline & Grünland & 34 & 0,089 & 0,072 & 0,074 & 0,099 & 0,073 \\
\hline \multirow[t]{2}{*}{ Sandiger Lehm } & Ackerland & 84 & 0,311 & 0,279 & 0,326 & 0,349 & 0,300 \\
\hline & Grünland & 34 & 0,080 & 0,065 & 0,083 & 0,097 & 0,06 \\
\hline \multirow[t]{2}{*}{ Lehm (ÖH) } & Ackerland & 86 & 0,389 & 0,354 & 0,415 & 0,446 & 0,394 \\
\hline & Grünland & 34 & 0,101 & 0,083 & 0,111 & 0,126 & 0,102 \\
\hline \multirow[t]{2}{*}{ Lehm (Marsch) } & Ackerland & 86 & 0,356 & 0,343 & 0,340 & 0,398 & 0,287 \\
\hline & Grünland & 34 & 0,076 & 0,096 & 0,079 & 0,102 & 0,064 \\
\hline Niedermoor & Grünland & 28 & 0,101 & 0,120 & 0,093 & 0,115 & 0,078 \\
\hline Hochmoor & Grünland & 11 & 0,043 & 0,076 & 0,037 & 0,052 & 0,033 \\
\hline
\end{tabular}

ÖH: Östliches Hügelland; Ein- und Austräge in $\mathrm{kg} \mathrm{P} / \mathrm{ha}$

${ }^{\text {a }}$ Niederschlag in $\mathrm{mm}$

nario weist nur bei den Lehmstandorten höhere P-Austräge im Vergleich zum Standort Erfde auf. Auf den sandigeren Standorten wird weniger P-Austrag simuliert als im Standort Lensahn. Dies kann damit begründet werden, dass im Referenzszenario im Durchschnitt der Jahre in den vegetationslosen und damit austragssensiblen Wintermonaten weniger Niederschlag berechnet wird als im Klimastandort Erfde. B1 weist durchgängig für alle Bodenarten die höchsten P-Austräge im Sickerwasser auf. Die Resultate für A1B sind mit den Ergebnissen des Referenzszenarios vergleichbar. Ausnahme sind die Sandstandorte, die höhere P-Austräge aufweisen. Die Zunahme der Niederschläge in den Wintermonaten führt zu diesem Ergebnis. Anders als bei lehmigeren Standorten versickert das Bodenwasser kaum rasch in größere Tiefe.

Die N-Einträge und -austräge der sandigen Standorte (Sand, Sand über Lehm und lehmiger Sand) weisen die höchsten N-Verluste mit dem Sickerwasser bei einem Flurabstand von 0,5 $\mathrm{m}$ auf (Tabelle 8). Die verminderte Pflanzenaufnahme führt bei niedrigen Grundwasserflurabständen zu erhöhten Austrägen. Anders als bei lehmigeren Standorten bei gleichem Flurabstand wird N weniger durch Denitrifikation emittiert. Bei Sand über Lehm und lehmigem Sand sind die Austräge in den Flurabstandsstufen nahezu gleich. Denn bei Trockenheit wird gespeichertes Bodenwasser aus tieferen Bodenschichten nachgeliefert, das Niederschlagswasser kann gut gespeichert werden, und eine gute Durchlüftung verhindert Staunässe. Die lehmigen Standorte zeigen höhere N-Austräge im Sickerwasser bei größeren Grundwasserflurabständen. Bodenarten mit niedrigen kF-Werten behindern bei Trockenheit eine schnelle Nachlieferung von Wasser aus tieferen Bodenschichten und reduzieren die N-Aufnahme der Pflanzen. Gleichzeitig behindert die N-Aufnahme durch die Pflanzen. Die Denitrifikationsrate der lehmigen Standorte beträgt bei Flurabständen zwischen $0,25 \mathrm{~m}$ und $0,5 \mathrm{~m}$ über $60 \mathrm{~kg} \mathrm{~N} / \mathrm{ha}$. Die N-Gesamtverluste sind auf lehmigen Standorten geringen Flurabstandes höher als bei größerem Flurabstand. Im B1-Szenario werden die höchsten Austräge durchgängig für alle Grundwasserflurabstandsstufen berechnet.

Die P-Austräge sind höher, wenn der Grundwasserflurabstand gering, d. h. der Grundwasserstand hoch ist (Tabelle 9). Das Szenario B1 weist auch hier die höchsten Austräge auf.

Die Ammonium-Auswaschung unterhalb der Pflanzenwurzelzone spielt bei der Betrachtung der Gesamt-N-Austräge mit dem Sickerwasser eine untergeordnete Rolle (Tabelle 10). Das Ammonium-Ion wird an den Austauschern des Bodens sorbiert oder aber von der Pflanze aufgenommen. Die Ammonium-Austräge im Sickerwasser sind bei der in WASMOD eingestellten Bilanzierungstiefe von $1 \mathrm{~m}$ mit max. ca. $9 \mathrm{~g} / \mathrm{ha}$ (Hochmoorstandorte) im Vergleich zu den frei beweglichen $\mathrm{NO}_{3}$-N-Austrägen gering.

Bei der mikrobiellen Denitrifikation wird unter anaeroben Bedingungen Nitrat oder Nitrit zu nitrosen Gasen reduziert. Schlecht belüftete Böden fördern somit die Denitrifikation. Die Denitrifikation und die Ausgasung bilden die Summe der gasförmigen N-Verluste der Landschaft. Die gasförmigen N-Verluste weisen auf den sandigen Standorten erwartungsgemäß kaum Denitrifikationsverluste auf (Tabelle 11). Bei den lehmigen Standorten können die Denitrifikationsverluste auf $41 \mathrm{~kg} \mathrm{~N} / \mathrm{ha}$ im Jahr ansteigen. Diese schlecht belüfteten Böden begünstigen insbesondere bei niedrigen Grundwasserflurabständen die Umwandlung des Nitrats oder Nitrits zu nitrosen Gasen. In den Klimaszena- 
Tabelle 8 N-Ein- und -Austräge bei verschiedenen Grundwasserflurabständen

\begin{tabular}{|c|c|c|c|c|c|c|c|}
\hline \multirow[b]{2}{*}{ Bodenart } & \multirow[b]{2}{*}{ Grundwasserflurabstand } & \multirow[b]{2}{*}{ Eintrag } & Erfde $(835)^{a}$ & Lensahn $(705)^{a}$ & Referenz $(838)^{a}$ & B1 $(934)^{a}$ & A1B $(876)^{2}$ \\
\hline & & & \multicolumn{5}{|c|}{$\operatorname{Austrag}\left(\mathrm{NO}_{3}-\mathrm{N}\right)$} \\
\hline \multirow[t]{3}{*}{ Sand } & $0,50 \mathrm{~m}$ & 133 & 80 & 71 & 81 & 86 & 78 \\
\hline & $1,00 \mathrm{~m}$ & 133 & 61 & 51 & 64 & 71 & 62 \\
\hline & $2,00 \mathrm{~m}$ & 133 & 66 & 58 & 69 & 74 & 66 \\
\hline \multirow[t]{3}{*}{ Sand über Lehm } & $0,50 \mathrm{~m}$ & 141 & 79 & 69 & 80 & 86 & 78 \\
\hline & $1,00 \mathrm{~m}$ & 141 & 75 & 65 & 75 & 82 & 74 \\
\hline & $2,00 \mathrm{~m}$ & 141 & 78 & 69 & 79 & 85 & 77 \\
\hline \multirow[t]{3}{*}{ Lehmiger Sand } & $0,50 \mathrm{~m}$ & 149 & 79 & 69 & 83 & 90 & 79 \\
\hline & $1,00 \mathrm{~m}$ & 149 & 72 & 62 & 75 & 82 & 72 \\
\hline & $2,00 \mathrm{~m}$ & 149 & 78 & 69 & 80 & 85 & 76 \\
\hline \multirow[t]{3}{*}{ Sandiger Lehm } & $0,50 \mathrm{~m}$ & 161 & 68 & 58 & 73 & 81 & 71 \\
\hline & $1,00 \mathrm{~m}$ & 161 & 69 & 60 & 73 & 78 & 67 \\
\hline & $2,00 \mathrm{~m}$ & 161 & 72 & 64 & 75 & 82 & 73 \\
\hline \multirow[t]{4}{*}{ Lehm (ÖH) } & $0,25 \mathrm{~m}$ & 169 & 57 & 52 & 59 & 64 & 58 \\
\hline & $0,50 \mathrm{~m}$ & 169 & 65 & 56 & 69 & 77 & 67 \\
\hline & $1,00 \mathrm{~m}$ & 169 & 73 & 62 & 75 & 80 & 71 \\
\hline & $2,00 \mathrm{~m}$ & 169 & 72 & 62 & 73 & 81 & 71 \\
\hline \multirow[t]{4}{*}{ Lehm (Marsch) } & $0,25 \mathrm{~m}$ & 169 & 67 & 55 & 66 & 74 & 64 \\
\hline & $0,50 \mathrm{~m}$ & 169 & 72 & 57 & 75 & 83 & 70 \\
\hline & $1,00 \mathrm{~m}$ & 169 & 72 & 62 & 75 & 80 & 71 \\
\hline & $2,00 \mathrm{~m}$ & 169 & 88 & 81 & 90 & 96 & 86 \\
\hline \multirow[t]{3}{*}{ Niedermoor } & $0,25 \mathrm{~m}$ & 100 & 41 & 37 & 42 & 43 & 39 \\
\hline & $0,50 \mathrm{~m}$ & 100 & 46 & 40 & 47 & 49 & 45 \\
\hline & $1,00 \mathrm{~m}$ & 100 & 55 & 47 & 56 & 56 & 50 \\
\hline \multirow[t]{2}{*}{ Hochmoor } & $0,25 \mathrm{~m}$ & 64 & 23 & 21 & 22 & 23 & 20 \\
\hline & $0,50 \mathrm{~m}$ & 64 & 27 & 24 & 26 & 27 & 25 \\
\hline
\end{tabular}

ÖH: Östliches Hügelland; Ein- und Austräge in kg N/ha

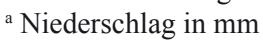

rien B1 und A1B sind die höchsten Denitrifikationsverluste $\mathrm{zu}$ verzeichnen.

Die Mineralisierung ist von großer Bedeutung für die Freisetzung von Haupt- und Spurennährstoffen beim Um- und Abbau in und auf dem Boden befindlicher organischer Stoffe. Die Mineralisation steigt mit dem Humusgehalt des Bodens und dem Wechsel von feuchten und trockeneren Phasen. Im Mittel aller berechneten 325 Merkmalskombinationen beträgt die Mineralisation $81 \mathrm{kgN} / \mathrm{ha}$ und Jahr. Bei dem Niedermoorstandort beträgt die Mineralisation mindestens $152 \mathrm{kgN} / \mathrm{ha}$ und Jahr (Klimastation Erfde). Der hohe Humusgehalt beeinflusst an diesen Standorten die Mineralisation beträchtlich. Mit höherem Grundwasserstand steigt die Mineralisationsrate bis auf durchschnittlich $215 \mathrm{~kg} \mathrm{~N} / \mathrm{ha} \mathrm{im} \mathrm{Jahr} \mathrm{(Lensahn),} \mathrm{da}$ feuchte und trockenere Perioden sich öfter über größere Bodenschichten abwechseln. Die höchsten Mineralisationsraten sind im Szenario A1B zu erwarten. Der hohe Temperaturanstieg bei gleichzeitiger Trockenheit im Sommer führt zu diesem Ergebnis. Das Szenario B1 weist zwar im Vergleich zum Referenzszenario ebenfalls erhöhte Temperaturen auf, jedoch sind aufgrund der hohen Niederschläge weniger Trockenperi- oden zu verzeichnen, sodass eine Abnahme der Mineralisationsleitung auf diesen Standorten berechnet wurde.

\section{Diskussion}

Sowohl die absolute Temperaturerhöhung als auch die $\mathrm{Zu}$ nahme der Temperaturvariabilität sowie die Verschiebung der Niederschlagsverteilung und damit verknüpft häufigere Hochwasserereignisse werden nicht ohne Folgen für die Funktionsweise von terrestrischen Ökosystemen bleiben. Deutliche Veränderungen der Pflanzenphänologie im $\mathrm{Zu}$ sammenhang mit Klimaveränderungen wurden nachgewiesen. Die Veränderung der Pflanzenphänologie beeinflusst den Wasser- und Stofffluss an den Grenzflächen der Pflanzen zur Atmosphäre und zum Boden. Darüber hinaus hat eine Veränderung der Phänologie von Nutzpflanzen agrarökonomische Bedeutung (z.B. Anbauzeiten, Sortenwahl, Bewirtschaftung, Absatz).

Neben dem Anstieg der Durchschnittswerte von Temperatur und Niederschlag wird sich voraussichtlich vor allem 
Tabelle 9 P-Einträge und -Austräge bei verschiedenen Grundwasserflurabständen

\begin{tabular}{|c|c|c|c|c|c|c|c|}
\hline & & & Erfde $(835)^{\mathrm{a}}$ & Lensahn $(705)^{a}$ & Referenz $(838)^{a}$ & B1 $(934)^{a}$ & A1B $(876)^{a}$ \\
\hline Bodenart & Grundwasserflurabstand & Eintrag & & & Austrag & & \\
\hline \multirow[t]{3}{*}{ Sand } & $0,50 \mathrm{~m}$ & 39 & 0,104 & 0,111 & 0,075 & 0,119 & 0,086 \\
\hline & $1,00 \mathrm{~m}$ & 39 & 0,066 & 0,064 & 0,056 & 0,073 & 0,064 \\
\hline & $2,00 \mathrm{~m}$ & 39 & 0,055 & 0,042 & 0,041 & 0,061 & 0,053 \\
\hline \multirow[t]{3}{*}{ Sand über Lehm } & $0,50 \mathrm{~m}$ & 40 & 0,374 & 0,279 & 0,179 & 0,224 & 0,196 \\
\hline & $1,00 \mathrm{~m}$ & 40 & 0,128 & 0,111 & 0,099 & 0,119 & 0,095 \\
\hline & $2,00 \mathrm{~m}$ & 40 & 0,108 & 0,096 & 0,087 & 0,110 & 0,089 \\
\hline \multirow[t]{3}{*}{ Lehmiger Sand } & $0,50 \mathrm{~m}$ & 49 & 0,222 & 0,197 & 0,179 & 0,212 & 0,154 \\
\hline & $1,00 \mathrm{~m}$ & 49 & 0,135 & 0,111 & 0,116 & 0,152 & 0,122 \\
\hline & $2,00 \mathrm{~m}$ & 49 & 0,116 & 0,089 & 0,099 & 0,136 & 0,110 \\
\hline \multirow[t]{3}{*}{ Sandiger Lehm } & $0,50 \mathrm{~m}$ & 52 & 0,239 & 0,224 & 0,239 & 0,270 & 0,234 \\
\hline & $1,00 \mathrm{~m}$ & 52 & 0,135 & 0,115 & 0,150 & 0,161 & 0,127 \\
\hline & $2,00 \mathrm{~m}$ & 52 & 0,113 & 0,086 & 0,120 & 0,131 & 0,092 \\
\hline \multirow[t]{4}{*}{ Lehm (ÖH) } & $0,25 \mathrm{~m}$ & 53 & 0,268 & 0,259 & 0,276 & 0,301 & 0,284 \\
\hline & $0,50 \mathrm{~m}$ & 53 & 0,264 & 0,231 & 0,274 & 0,298 & 0,270 \\
\hline & $1,00 \mathrm{~m}$ & 53 & 0,151 & 0,136 & 0,182 & 0,199 & 0,169 \\
\hline & $2,00 \mathrm{~m}$ & 53 & 0,134 & 0,094 & 0,145 & 0,164 & 0,104 \\
\hline \multirow[t]{4}{*}{ Lehm (Marsch) } & $0,25 \mathrm{~m}$ & 53 & 0,241 & 0,328 & 0,227 & 0,249 & 0,216 \\
\hline & $0,50 \mathrm{~m}$ & 53 & 0,181 & 0,189 & 0,213 & 0.249 & 0,139 \\
\hline & $1,00 \mathrm{~m}$ & 53 & 0,147 & 0,122 & 0,135 & 0,167 & 0,114 \\
\hline & $2,00 \mathrm{~m}$ & 53 & 0,135 & 0,099 & 0,114 & 0,166 & 0,106 \\
\hline \multirow[t]{3}{*}{ Niedermoor } & $0,25 \mathrm{~m}$ & 28 & 0,100 & 0,124 & 0,113 & 0,123 & 0,093 \\
\hline & $0,50 \mathrm{~m}$ & 28 & 0,110 & 0,158 & 0,084 & 0,110 & 0,072 \\
\hline & $1,00 \mathrm{~m}$ & 28 & 0,092 & 0,078 & 0,082 & 0,113 & 0,069 \\
\hline \multirow[t]{2}{*}{ Hochmoor } & $0,25 \mathrm{~m}$ & 11 & 0,039 & 0,093 & 0,043 & 0,057 & 0,036 \\
\hline & $0,50 \mathrm{~m}$ & 11 & 0,046 & 0,059 & 0,032 & 0,047 & 0,030 \\
\hline
\end{tabular}

ÖH: Östliches Hügelland; Ein- und Austräge in $\mathrm{kg} \mathrm{P} / \mathrm{ha}$

a Niederschlag in $\mathrm{mm}$

Tabelle 10 P-Einträge und -Austräge bei verschiedenen Grundwasserflurabständen

\begin{tabular}{|c|c|c|c|c|c|c|c|}
\hline & & & Erfde $(835)^{a}$ & Lensahn $(705)^{a}$ & Referenz $(838)^{a}$ & B1 $(934)^{a}$ & $\operatorname{A1B}(876)^{a}$ \\
\hline Bodenart & Nutzung & Eintrag & & & & & \\
\hline \multirow[t]{2}{*}{ Sand } & Ackerland & 148 & 0,75 & 0,62 & 0,70 & 0,82 & 0,72 \\
\hline & Grünland & 125 & 0,66 & 0,62 & 0,64 & 0,69 & 0,66 \\
\hline \multirow[t]{2}{*}{ Sand über Lehm } & Ackerland & 170 & 0,07 & 0,09 & 0,07 & 0,09 & 0,07 \\
\hline & Grünland & 125 & 0,06 & 0,08 & 0,06 & 0,08 & 0,06 \\
\hline \multirow[t]{2}{*}{ Lehmiger Sand } & Ackerland & 164 & 0,03 & 0,03 & 0,04 & 0,04 & 0,03 \\
\hline & Grünland & 140 & 0,40 & 0,34 & 0,37 & 0,46 & 0,41 \\
\hline \multirow[t]{2}{*}{ Sandiger Lehm } & Ackerland & 199 & 0,03 & 0,03 & 0,03 & 0,03 & 0,03 \\
\hline & Grünland & 140 & 0,22 & 0,19 & 0,21 & 0,25 & 0,21 \\
\hline \multirow[t]{2}{*}{ Lehm (ÖH) } & Ackerland & 215 & 0,00 & 0,00 & 0,00 & 0,00 & 0,00 \\
\hline & Grünland & 144 & 0,00 & 0,00 & 0,00 & 0,00 & 0,00 \\
\hline \multirow[t]{2}{*}{ Lehm (Marsch) } & Ackerland & 215 & 0,00 & 0,00 & 0,00 & 0,00 & 0,00 \\
\hline & Grünland & 144 & 0,07 & 0,08 & 0,07 & 0,08 & 0,07 \\
\hline Niedermoor & Grünland & 100 & 2,57 & 3,76 & 2,89 & 2,98 & 2,46 \\
\hline Hochmoor & Grünland & 64 & 5,56 & 8,71 & 5,93 & 5,99 & 5,77 \\
\hline
\end{tabular}

ÖH: Östliches Hügelland; N-Einträge in $\mathrm{kg} \mathrm{N} / \mathrm{ha}, \mathrm{NH}_{4}$-Austräge in $\mathrm{g} \mathrm{N} / \mathrm{ha}$

a Niederschlag in $\mathrm{mm}$ 
Tabelle 11 N-Einträge und Denitrifikation bei Grünlandnutzung

\begin{tabular}{|c|c|c|c|c|c|c|c|}
\hline & & & Erfde $(835)^{a}$ & Lensahn $(705)^{a}$ & Referenz $(838)^{a}$ & B1 $(934)^{a}$ & A1B $(876)^{a}$ \\
\hline Bodenart & Nutzung & Eintrag & \multicolumn{5}{|c|}{ Denitrifikation } \\
\hline Sand & Grünland & 125 & 1 & 1 & 1 & 1 & 1 \\
\hline Sand über Lehm & Grünland & 125 & 1 & 1 & 1 & 1 & 1 \\
\hline Lehmiger Sand & Grünland & 140 & 8 & 8 & 7 & 7 & 8 \\
\hline Sandiger Lehm & Grünland & 140 & 26 & 25 & 26 & 28 & 27 \\
\hline Lehm $(\mathrm{ÖH})$ & Grünland & 144 & 41 & 37 & 41 & 46 & 45 \\
\hline Lehm (Marsch) & Grünland & 144 & 26 & 25 & 27 & 31 & 30 \\
\hline Niedermoor & Grünland & 100 & 9 & 11 & 8 & 7 & 9 \\
\hline Hochmoor & Grünland & 64 & 5 & 9 & 5 & 4 & 6 \\
\hline
\end{tabular}

ÖH: Östliches Hügelland; Einträge und Denitrifikation in $\mathrm{kg} \mathrm{N} / \mathrm{ha}$

${ }^{a}$ Niederschlag in $\mathrm{mm}$

auch die raumzeitliche Variabilität dieser beiden Klimaelemente ändern. Dies hat voraussichtlich weitreichende Auswirkungen auf den Wasser- und Nährstoffhaushalt und damit direkt oder indirekt auf terrestrische und aquatische Ökosysteme und die Landnutzung, vor allem durch Landund Forstwirtschaft (Meiwes et al. 2007). Zusätzlich können der demografische Wandel, Änderungen der Landbewirtschaftung aufgrund sich wandelnder agrarökonomischer Rahmenbedingungen sowie steigender Flächenverbrauch für Siedlung und Verkehr die Wasser- und Stoffflüsse regional beeinflussen. Das kleinräumige Muster der Landnutzung und deren Änderung überlagern dabei die möglichen regionalen Auswirkungen des Klimawandels und können Effekte wie Abfluss und Nährstoffaustrag von Böden in die Fließgewässer verstärken oder mindern. Für eine verlässliche Prognose zukünftiger regionaler Entwicklungen des Wasser- und Nährstoffhaushaltes müssen folglich Auswirkungen der Klimaänderungen und die Effekte gleichzeitigen Landnutzungswandels kombiniert betrachtet werden.

Für Waldökosysteme ist zu erwarten, dass aufgrund von erhöhter Interzeption und Transpiration bei verminderten Niederschlägen während der Vegetationszeit Trockenstress vermehrt auftreten wird (Bréda et al. 2006). Dies erhöht die Disposition der Waldbestände für die Beeinträchtigung durch biotische Schädlinge (Bolte und Ibisch 2007). Für die Bewirtschaftung hat dies zu Folge, dass bisherige Anbauempfehlungen für Baumarten überprüft werden müssen. Beispielsweise ist eine deutliche Verkleinerung des Anbauareals der Fichte zu erwarten (Paar et al. 2005; Kölling et al. 2007).

Bislang weitgehend unberücksichtigt bei der Klimafolgenforschung bleibt der Stoffhaushalt der Böden, obwohl diese als regulatorisches Hauptkompartiment terrestrischer Ökosysteme neben dem Standortklima die zweite essenzielle Randbedingung der Primärproduktion sind. Für Waldböden ist durch Änderungen des Temperatur- und des Feuchteregimes mit Änderungen der Humusdynamik auszugehen, die weit reichende Folgen insbesondere für den Stickstoffund Säurehaushalt aufgrund starker Kopplungen mit den Umsätzen organischer Substanz erwarten lassen (Puhe und Ulrich 2001).

Die Ergebnisse dieser Arbeit können nicht anhand von Befunden vergleichbarer Untersuchungen diskutiert werden, denn solche gibt es bislang nicht. Die Befunde dieser pilothaften Berechnungen weisen zum einen darauf hin, dass der Klimawandel den Stoff- und Wasserhaushalt sowie die Stoffflüsse zwischen den Böden und der Atmosphäre sowie dem Grundwasser verändern wird. Die gefundenen Ergebnisse sind plausibel und entsprechen den Erwartungen. Mit Blick auf die Frage nach Anpassungen an den Klimawandel deuten die Simulationsergebnisse an, dass die Art der Landnutzung in einem durch die Bodenverhältnisse mit beeinflussten Rahmen Möglichkeiten zur Anpassung bietet. Dies ist insbesondere mit Blick auf die Umsetzung der EUWasserrahmenrichtlinie bedeutsam.

Vor diesem Hintergrund ist es eigentlich erstaunlich, dass die Modellierung von Prozessen im Boden in der Klimafolgenforschung bislang allenfalls eine Nebenrolle spielte. Schließlich sind Böden die regulatorischen Hauptkompartimente terrestrischer Ökosysteme, die mit der Atmosphäre und der Hydrosphäre in engen stofflichen und energetischen Wechselbeziehungen stehen. Simulationsmodelle sind in der Meteorologie weit entwickelt und im Wesentlichen anerkannt, in der Ökologie werden Modelle kontroverser diskutiert. Dabei wird bisweilen übersehen, dass sie in Kombination mit GIS räumlich konkretisierte Prognosen und Technologien (Was muss getan werden, um einen angestrebten Systemzustand zu ermöglichen?) liefern können (Schröder und Daschkeit 2003). Das in dieser Arbeit verwendete Modellsystem Wasmod wurde für sehr unterschiedlich ausgeprägte Boden-, Klima- und Nutzungsverhältnisse auf mehreren räumlichen Ebenen (Bodenprofil, Acker, Wassereinzugsgebiet) an Messdaten validiert. Eine besondere Bedeutung kam dabei dem Einfluss von Bewirtschaftungsmaßnahmen auf 
den Stoffaustrag aus Böden zu. Ausführliche Diskussionen und umfangreiche Literatur über weitere WASMOD-Anwendungen sind den Arbeiten von Fink und Kralisch (2005), Haluszczak et al. (1991), Kralisch et al. (2003, 2005), Müller et al. (2006) und Rinker (2001) zu entnehmen. Die in der vorliegenden Arbeit mit WASMOD berechneten der N- und P-Austräge bei steigenden Lufttemperaturen sind räumliche und zeitliche Prognosen. Vorhersagen sind Erweiterungen von räumlich und zeitlich punktuellen Stichproben auf andere Raum- und Zeitpunkte, also von Stichproben auf Grundgesamtheiten, deren verkleinertes Abbild die Stichproben sein sollen. Räumliche Generalisierung ist die Verallgemeinerung von Messungen an ausgewählten Orten auf solche ohne Messungen. Bei der zeitlichen Generalisierung werden Stichprobenereignisse eines status quo auf einen status post (Prognose) oder einen status ante (Epignose) bezogen. Die empirische Validität zeitlicher Prognosen wie die in dieser Arbeit kann erst dann geprüft werden, wenn der prognostizierte Zeitpunkt erreicht ist, also ex post. Die solcherart bestimmte Prognosequalität von WASMOD ist ausreichend (Fink und Kralisch 2005; Haluszczak et al. 1991; Kralisch et al. 2003, 2005; Müller et al. 2006; Rinker 2001).

\section{Schlussfolgerungen}

Die Berechnungen zeigen, dass die sich großräumig veränderten Energieflüsse auf die räumliche und zeitliche Struktur sowie die Amplituden der Ausprägung atmosphärischer Merkmale auswirken. Dies zeigt sich besonders deutlich an den Lufttemperaturen und den Niederschlägen. Diese beiden Klimaelemente sind entscheidende Regelgrößen für die Stoffhaushalte von Böden terrestrischer Ökosysteme. Die Folgen ihrer empirisch belegten und ihrer prognostizierten Veränderung können für den N- und P-Austrag aus Böden Schleswig-Holsteins mit dem Modellsystem WASMOD berechnet werden. Wegen der umfassenden Validierung von WASMOD in der Ökosystemforschung, in Fallstudien sowie im Umwelt-Monitoring kann davon ausgegangen werden, dass der Wasser- und Stoffhaushalt der Böden zuverlässig modelliert wird. Grundlagenforschung in Form der Ökosystemforschung erweist sich insofern als lohnend, als man dadurch jetzt in der Lage ist, validierte Modelle wie Wasmod für Prognosen des Stoffhaushalts in Böden unter veränderten Klimabedingungen berechnen zu können. WASMOD könnte auch einen Beitrag für die im Zusammenhang mit dem Klimawandel relevante Prognose des Risikos der Ausbreitung von Krankheiten liefern, deren Überträger sich im warm-feuchten Milieu ausbreiten (Maier et al. 2003; Takken et al. 2005; WHO 2004). Hierbei ist die Bodenfeuchte bedeutsam, die anhand der Daten der Reichsbodenschätzung mit WASMOD kleinräumig differenziert berechnet werden kann.

\section{Empfehlungen und Ausblick}

Grundlagenforschung im Schnittfeld von Ökologie und Umweltmedizin wäre die beste Vorbereitung auf Umweltkrisen und sollte fortentwickelt werden (Markert et al. 2008; Rüdel et al. 2007). Hierbei muss jedoch streng darauf geachtet werden, dass von vornherein ein stringentes statistisches Design entworfen und realisiert wird. Die Steigerung der umweltwissenschaftlichen Prognosefähigkeit setzt ferner voraus, dass einmal erarbeitetes Wissen in Form von Daten endlich professionell nach dem Vorbild des Bibliothekswesens professionell dokumentiert, archiviert und miteinander vernetzt wird (Bill 2005; Schröder et al. 2008).

Die Auswirkungen des Klimawandels auf den Stoffhaushalt in Böden sollen mit WASMOD bundesweit regionalisiert und mit Befunden aus dem Bio-Monitoring (de Bryn et al. 2009; Schröder et al. 2008; Wappelhorst et al. 2000) verknüpft werden. Hierfür werden auf der Grundlage einer landschaftsökologischen Gliederung (Hornsmann et al. 2008; Schröder und Schmidt 2000) sowie anhand von Klimadaten und pflanzenphänologischen Daten des Deutschen Wetterdienstes und der internationalen phänologischen Gärten (Schröder et al. 2005) repräsentative Modellierungsräume ausgewählt und untersucht. Die Befunde sollten in einer WebGIS-Anwendung (Kleppin et al. 2008; Schmidt et al. 2009) umfassend dokumentiert und für die weitere Nutzung zur Verfügung gestellt werden. Weiterhin soll mit WASMOD der Wasserhaushalt an der Bodenoberfläche unter veränderten Klimabedingungen simuliert werden, was für eine prädiktive Kartierung des Risikos vektorassoziierter Krankheiten wesentlich ist (Schröder et al. 2007).

\section{Literatur}

Bill R (2005) DFG-Rundgespräch „Geowissenschaftliche Informationsportale", Hannover, 21./22. Februar 2005. Rostock

Bolte A, Ibisch PL (2007) Neun Thesen zu Klimawandel, Waldbau und Waldnaturschutz. Wald 11:572-576

Bréda N, Huc R, Granier A, Dreyer E (2006) Temperate forest trees and stand under severe drought: a review of ecophysiological responses, adaption processes and long-term consequences. Ann Forest Sci 63:625-644

de Bruyn U, Linders H-W, Mohr K (2009) Epiphytische Flechten im Wandel von Immissionen und Klima - Ergebnisse einer Vergleichskartierung 1989/2007 in Nordwestdeutschland. Umweltwiss Schadst Forsch 21:63-75

EEA (2004) Impacts of Europe's changing climate. An indicator-based assessment. EEA Report 2:1-100

Ellenberg H, Fränzle O, Müller P (1978) Ökosystemforschung im Hinblick auf Umweltpolitik und Umweltplanung. Umweltforschungsplan des Bundesministeriums des Inneren, FKZ 78-10104005. Berlin

Fränzle O (1998) Grundlagen und Entwicklung der Ökosystemforschung. In: Fränzle O, Müller F, Schröder W (Hrsg) Handbuch der Umweltwissenschaften. Grundlagen und Anwendungen der Ökosystemforschung, 3. ErgLfg, Kap II-2.1:1-24. ecomed, Landsberg am Lech München Zürich 
Fränzle O, Jensen-Huß K, Daschkeit A, Hertling T, Lüschow R, Schröder W (1993) Grundlagen zur Bewertung der Belastung und Belastbarkeit von Böden als Teilen von Ökosystemen. UBA-Texte 59/93, Berlin

Fränzle O, Kappen L, Blume H-P, Dierssen K (eds) (2008) Ecosystem organization of a complex landscape. Long-term research in the Bornhöved lake district, Germany. Ecological Studies 202. Springer, Berlin, 392 Seiten

Fink M, Kralisch S (2005) Estimating the importance of topological routing for modelling water and nitrogen transport at catchment scale. In: Zerger A, Argent RM (eds) MODSIM 2005 International Congress on Modelling and Simulation. Modelling and Simulation Society of Australia and New Zealand, Dezember 2005, pp 2686-2692

Haluszczak S, Vetter L, Schröder W (1991) Dehydrogenaseaktivität in konventionell und biologisch bewirtschafteten Böden unterschiedlicher Nutzung. Schr Naturwiss Ver Schleswig-Holstein 61:55-80

Hornsmann I, Schmidt G, Schröder W (2008) Berechnung einer landschaftsökologischen Raumgliederung Europas. Umweltwiss Schadst Forsch 20:25-35

IPCC (Intergovernmental Panel on Climate Change) (2001) Climate change. The scientific basis. University Press, Cambrigde, UK

Kleppin L, Schröder W, Pesch R, Schmidt G (2008) Entwicklung und Erprobung einer Metadaten- und WebGIS-Applikation für das Expositionsmonitoring mit Moosen in Deutschland. Ein Beitrag zum LTER-Netzwerk. Umweltwiss Schadst Forsch 20: 38-48

Kölling C, Zimmermann L, Walentowski H (2007) Klimawandel: Was geschieht mit Buche und Fichte? Wald 11:584-588

Kralisch S, Fink M, Flügel WA, Beckstein C (2003) A neural network approach for the optimization of watershed management. Envrion Model Softw 18:815-823

Kralisch S, Fink M, Beckstein C (2005) Neural Network Based Sensitivity Analysis of Natural Resource Models. In: Zerger A, Argent RM (eds) MODSIM 2005 International Congress on Modelling and Simulation. Modelling and Simulation Society of Australia and New Zealand, pp 2498-2504

LWK-SH (Landwirtschaftskammer Schleswig-Holstein) (2001a) Marktfrucht-Report 2001. Ergebnisse und Analysen aus der Unternehmensberatung für Marktfruchtbetriebe in Schleswig-Holstein. Betriebswirtschaftliche Mitteilungen NR. 550/551, Kiel, S 1-57

LWK-SH (2001b) Ökologisch-Ökonomische Auswirkungen des Integrierten Landbaus. Betriebswirtschaftliche Mitteilungen NR. 552/553, Kiel, S 1-102

LWK-SH (2001c) Ergebnisse der Rinderspezialberatung, Abteilung Betriebswirtschaft und Beratung. Betriebswirtschaftliche Mitteilungen NR. 554/555, Kiel, S 1-125

LWK-SH (2003) Richtwerte für die Düngung. Kiel

Maier WA, Grunewald J, Habedank B, Hartelt K, Kampen H, Kimmig P, Naucke T, Oehme R, Vollmer A, Schöler A, Schmitt C (2003) Mögliche Auswirkungen von Klimaveränderungen auf die Ausbreitung von primär humanmedizinisch relevanten Krankheitserregern über tierische Vektoren sowie auf die wichtigen Humanparasiten in Deutschland. Climate Change 05/03, Berlin, 386 Seiten

Markert B, Wünschmann S, Fränzle S, Wappelhorst O, Weckert V, Breulmann G, Djingova R, Herpin U, Lieth H, Schröder W, Siewers U, Steinnes E, Wolterbeek B, Zechmeister H (2008) On the road from biomonitoring to human health aspects - monitoring atmospheric heavy metal deposition by epiphytic/epigeic plants: present status and future needs. Int J Environ Pollut 32:486-498

Matthies M, Klasmeier J, Heß O (2006) GIS-basietre Modellierung von Gewässerimmissionen. Umweltwiss Schadst Forsch 18:102-109

Meiwes KJ, Badeck F-W, Beese F, Berthold D, Einert P, Jochheim H, Kallweit R, Konopatzky A, Lasch P, Meesenburg H, Puhlmann M, Raspe S, Schulte-Bisping H, Schulz C, Suckow F (2007) Kohlenstoffumsatz in Waldökosystemen und deren Böden: Parame- trisierung, Kalibrierung und Validierung von Modellen. Wald 20:1075-1077

MLLT (Ministerium für ländliche Räume, Landesplanung, Landwirtschaft und Tourismus des Landes Schleswig Holstein) (2002) Agrarreport Schleswig-Holstein. Kiel, S 1-189

Müller F, Schrautzer J, Reiche EW, Rinker A (2006) Ecosystem based indicators in retrogressive successions of an agricultural landscape. Ecol Ind 6:63-82

Paar U, Schmidt M, Schönfelder E, Mues V, Scheler B, Eichhorn J (2005) Kartenbezogene Grundlagen der Waldentwicklung unter dem Einfluss von Klimaänderungen. Abschlussbericht InKlim 2012 Baustein II. Hessen-Forst. Servicestelle für Forsteinrichtung, Information und Versuchswesen, Hannoversch Münden, $24 \mathrm{~S}$

Puhe J, Ulrich B (2001) Global climate change and human impacts on forest ecosystems. Ecological Studies 143. Springer, Berlin, 592 Seiten

Reiche EW (1991) Entwicklung, Validierung und Anwendung eines Modellsystems zur Beschreibung und flächenhaften Bilanzierung der Wasser- und Stickstoffdynamik in Böden. Kieler Geograph Schr 79:1-150

Rinker A (2001) Beschreibung der Wasser- und Stoffhaushaltsdynamik devastierter Flächen mit dem Simulationsmodell WASMOD am Beispiel des Braunkohlentagesbergbaus Espenhain. Diss. Fak. Geowiss., Geotechnik und Bergbau, TU Bergakademie Freiberg, Freiberg

Rüdel H, Bester K, Eisenträger A, Franzaring J, Haarich M, Köhler J, Körner W, Oehlmann J, Paschke A, Ricking M, Schröder W, Schröter-Kermani Ch, Schulze T, Schwarzbauer J, Theobald N, von der Trenck Th, Wagner G, Wiesmüller G A (Arbeitskreis Umweltmonitoring in der GDCh-Fachgruppe Umweltchemie und Ökotoxikologie) (2007) Stoffbezogenes Umweltmonitoring. In: Wichmann H-E, Schlipköter H-W, Füllgraf G (Hrsg) Handbuch der Umweltmedizin. Loseblattwerk, 37, Bd. 2, Kap. IV-7. Erg. Lfg., ecomed MEDIZIN, Landsberg, S 1-48

Schmidt G, Aden C, Kleppin L, Pesch R, Schröder W (2009) Integration of long-term environmental data by the example of the UNECE Heavy Metals in Mosses Survey in Germany: Application of a WebGIS-based metadata system. In: Müller F, Klotz S, Schubert $\mathrm{H}$ (Hrsg) Long-term ecological research - Between theory and application. Springer, Berlin (im Druck)

Schröder W, Daschkeit A (2003) Umweltwissenschaft als Prototyp interdisziplinärer Forschung. In: Fränzle O, Müller F, Schröder W (Hrsg) Handbuch der Umweltwissenschaften. Grundlagen und Anwendungen der Ökosystemforschung, Kap. II-2.5 (9. ErgLfg). ecomed, Landsberg am Lech München Zürich, S 1-32

Schröder W Schmidt G (2000) Raumgliederung für die Ökologische Umweltbeobachtung des Bundes und der Länder. Umweltwiss Schadst Forsch 12:237-243

Schröder W, Schmidt G, Hasenclever J (2005) Korrelation meteorologischer und phänologischer Indikatoren des Klimawandels? Statistische Analyse am Beispiel Baden-Württembergs. Umweltwiss Schadst Forsch 17:94-105

Schröder W, Bast H, Pesch R, Schmidt G, Kiel E (2007) Flächenhafte Modellierung der potenziellen Reproduktionsrate des MalariaErregers Plasmodium vivax in Anopheles atroparvus auf Grundlage gemessener und prognostizierter Lufttemperaturen in Niedersachsen. Umweltwiss Schadst Forsch 19:115-122

Schröder W, Hornsmann I, Pesch R, Schmidt G, Fränzle S, Wünschmann S, Heidenreich H, Markert B (2008) Moosmonitoring als Spiegel der Landnutzung? Stickstoff- und Metallakkumulation in Moosen zweier Regionen Mitteleuropas. Umweltwiss Schadst Forsch 20:62-74

StA-HHSH (Statistisches Amt für Hamburg und Schleswig-Holstein) (2004a) Die Bodennutzung in Schleswig-Holstein in landwirtschaftlichen Betrieben. Endgültiges Ergebnis. Kiel, S 1-11 
StA-HHSH (2004b) Statistisches Jahrbuch 2004, Kap. 11, Landwirtschaft. Kiel, S 1-301

StA-HHSH (2004c) Die Bodennutzung in Schleswig-Holstein 2004. Kiel, (C I1-j/04)

StA-HHSH (2004d) Bodennutzung und Ernte in Schleswig-Holstein 2003. Kiel, (C I/C I1-j/03 S)

StA-HHSH (2005a) Agrarstruktur in Schleswig-Holstein 2003. Ausgewählte Strukturdaten für Betriebe mit ökologischem Landbau nach Kreisen und Naturräumen. Ergebnisse der Agrarstrukturerhebung, Statistischer Bericht, 19. Mai 2005, Kiel, S 1-20

StA-HHSH (2005b) Bodennutzung und Ernte in Schleswig-Holstein 2003. Statistischer Bericht, 16. September 2004, Kiel, S 1-87
StA-HHSH (2005c) Ernteberichterstattung über Feldfrüchte und Grünland Schleswig-Holstein. Statistischer Bericht, CII 1-m9/05S, 6. Oktober 2005, Kiel

Takken W, Martens P, Bogers (eds) (2005) Environmental change and malaria risk. Global and local implications. Springer, Dordrecht

Wappelhorst O, Korhammer S, Leffler US, Markert B (2000) Ein Moosbiomonitoring zur Ermittlung atmosphärischer Elementeinträge in die Euroregion Neiße (D, PL, CZ). Umweltwiss Schadst Forsch 12:191-200

WHO: World Health Organization (2004) Using Climate to predict infectious disease outbreaks. A Review. WHO/SDE/OEH/04.01, Geneva 